\title{
Sensitivity Analysis of Multicarrier Digital Pre-distortion/ Equalization Techniques for Non-linear Satellite Channels
}

\author{
R. Piazza ${ }^{1}$ and M. R. Bhavani Shankar ${ }^{2}$ \\ SnT, University of Luxembourg, Luxembourg, Luxembourg, L-2721 \\ E. Zenteno ${ }^{3}$ and D. Rönnow ${ }^{4}$ \\ University of Gävle, Gävle, Sweden, 801-76 \\ K. Liolis ${ }^{5}$ and F. Zimmer ${ }^{6}$ \\ SES TechCom S.A., Betzdorf, Luxembourg, L-6815 \\ M. Grasslin ${ }^{7}$ and T. Berheide ${ }^{8}$ \\ Steinbeis Transfer Centre for Space, Gäufelden, Germany, 71126 \\ and \\ S. Cioni ${ }^{9}$ \\ ESA/ESTEC, Noordwijk, Netherlands, 2200 AG
}

$\begin{array}{lll}A C I & = & \text { Adjacent Channel Interferences } \\ A P S K & = & \text { Amplitude Phase Shift Key } \\ B E R & = & \text { Bit Error Rate } \\ D P D & = & \text { Digital Predistortion } \\ D T H & = & \text { Direct To Home } \\ E Q & = & \text { EQualization } \\ F I R & = & \text { Finite Impulse Response } \\ G W & = & \text { Gate Way } \\ H P A & = & \text { High Power Amplifier } \\ I B O & = & \text { Input Back-Off } \\ I M U X & = & \text { Input } \\ I S I & = & \text { Inter-Symbol Interference } \\ L-T W T A & = & \text { Linearized Travelling Wave Tube Amplifier } \\ \text { LS } & = & \text { Least Squares } \\ \text { OBO } & = & \text { Output Back-Off } \\ \text { OMUX } & = & \text { Output } \\ \text { RLS } & = & \text { Recursive Least Squares } \\ \text { TD } & = & \text { Total Degradation }\end{array}$

1 Doctoral Student, SnT, roberto.piazza@uni.lu, non-member

Research Scientist, SnT, Bhavani.Shankar@uni.lu, non-member

3 Doctoral Student, University of Gävle, Efrain.Zenteno@hig.se, non-member

4 Professor, University of Gävle, Daniel.Ronnow@ hig.se, non-member

5 Space System Engineer, SES, Konstantinos.Liolis@ ses.com, non-member

6 Senior Manager, SES, Frank.Zimmer@ ses.com, non-member

7 Senior Scientist, TZR, graesslin@tz-raumfahrt.de, non-member

8 Physicist, TZR, tobias.berheide@ @z-raumfahrt.de, non-member

9 Communications Systems Engineer, TEC-ETC, stefano.cioni@esa.int, non-member

* This work was done under the ESA ARTES 5.1 project "On Ground Multicarrier Digital Equalization/Predistortion Techniques for Single or Multi Gateway Applications" (APEXX) - ESA ESTEC Contract No.: 4000105192/12/NL/AD. Responsibility for the presented content resides in the authors and organizations that prepared it. 


\begin{abstract}
On-board joint power amplification of multiple-carrier DVB-S2 signals using a single High-Power Amplifier (HPA) is an emerging configuration that aims to reduce flight hardware and weight. However, effects specific to such a scenario degrade power and spectral efficiencies with increased Adjacent Channel Interference caused by non-linear characteristic of the HPA and power efficiency loss due to the increased Peak to Average Power Ratio (PAPR). The paper studies signal processing techniques that counteract for both aspects enabling efficient multicarrier on board amplification. Digital pre-distortion (DPD) at the gateway and equalization (EQ) at the User Terminal are the designed avenues of attack to mitigate the non-linear effects and improve power as well as spectral efficiencies. This paper builds on our initial work in Ref.1 that performed a survey on the adaptability of various compensation techniques to the considered scenario and short-listed the most favourable ones. Preliminary performance analysis of these techniques was undertaken with Signal to Interference Ratio (SINR) as the figure of merit. The contribution of this work over Ref. 1 includes : (a) a full DVB-S2 chain is simulated with the chosen techniques with Total Degradation (TD) as the figure of merit and (b) a sensitivity analysis is performed to study the dependence of the pre-distortion/ equalization performance ( measured as TD) on the channel parameters.
\end{abstract}

\title{
I. Introduction
}

Today's satellite communication scenarios typically imply that the uplinked signal is amplified and channelized in a transparent satellite transponder. Power efficient on-board amplification brings non-linear distortions limiting the usage of spectrally efficient modulation schemes. In future scenarios increase in data rates will require higher spectral efficiencies. Further, joint amplification of multiple-carrier DVB-S2 signals using a single High-Power Amplifier (HPA) is envisaged due to sharing of satellite capacity among different links and to meet power/mass requirements. The non-linear effects are even more prominent in this scenario due to onset of intermodulation products causing adjacent channel interference (ACI). A significant guard-band between the carriers may be needed in order to avoid ACI, thereby reducing spectral efficiency. Additionally, use of multiple carriers leads to high peak to average power ratios; this increases the back-off leading an amplification efficiency loss. Moreover, on-board channelization filters (IMUX/ OMUX) introduce inter-symbol interference (ISI), which further degrades the performance.

In order to mitigate the nonlinear distortion, significant back off is required, leading to power efficiency loss. This motivates the need to study techniques to improve power and spectral efficiencies. These techniques - digital predistortion (DPD) at the gateway and equalization (EQ) at the User Terminal- have been well documented in literature for single carrier per HPA ${ }^{2-7}$ and only a few results are available for multicarrier case ${ }^{8-10}$.

A study of DPD and EQ for multiple carrier satellite channels was undertaken and preliminary results were recently reported ${ }^{1}$ A survey on the adaptability of various compensation techniques to the considered scenario and a shortlisting of the most favourable ones were reported ${ }^{1}$. Preliminary performance analysis of these techniques was undertaken with Signal to Interference Ratio (SINR) as the figure of merit. The contribution of this work over Ref. 1 includes

1. A full DVB-S2 chain is simulated with the chosen techniques using the Total Degradation (TD) as the figure of merit

2. A detailed study of the effect of different channel parameters (linearized/ non-linearized HPA, IMUX/ OMUX filters, roll-off) and carrier configurations (modulation/ coding, carrier spacing and bandwidths) on the degradation in the multicarrier channel

3. A study of the sensitivity of the channel performance (measured as TD) to these key channel parameters also with respect to predistortion/equalization techniques..

The organization of the paper is as follows: we describe: the scenario supporting multiple carriers per HPA, the non-linear channel model and its implications to multiple carrier transmissions in Section II, enhancement 
techniques DPD and EQ in Section III and their performance analysis in Section IV. Conclusions are drawn in Section V.

\section{Multiple Carrier Satellite Transmissions}

\section{A. Scenario}

Figure 1 illustrates the addressed satellite system scenario, which refers to a multicarrier satellite channel where independent channels are uplinked to a transparent satellite. A gateway transmits a broadcast or broadband forward link carrier, typically DVB-S2 or similar signals, to a number of receivers. The considered frequency bands are mainly Ka-band and Ku-band frequencies for broadcast or broadband fixed satellite services (BSS/FSS) applications.

On board joint filtering and amplification takes place before the signals are downlinked to ground receivers. As described in Ref. 1 joint on board filtering and amplification of the stream of carriers allows a significant saving in hardware complexity and weight. Each carrier channel is assumed to be compliant with DVB-S2 standard.

On ground techniques are developed in order to increase power and spectral efficiency of the transmission. Predistortion is performed at the transmitting gateway jointly over all the independent carriers. Channel knowledge is obtained from dedicated user terminals that provide periodic loopback signals. On the other hand, equalization at the user terminal is limited to single carrier processing due to the nature of the DVB-S2 broadcast service. Equalization parameters can be extracted with training symbols or data aided techniques. Thus, adaptation to possible fast channel variations is tracked by receivers' equalization.

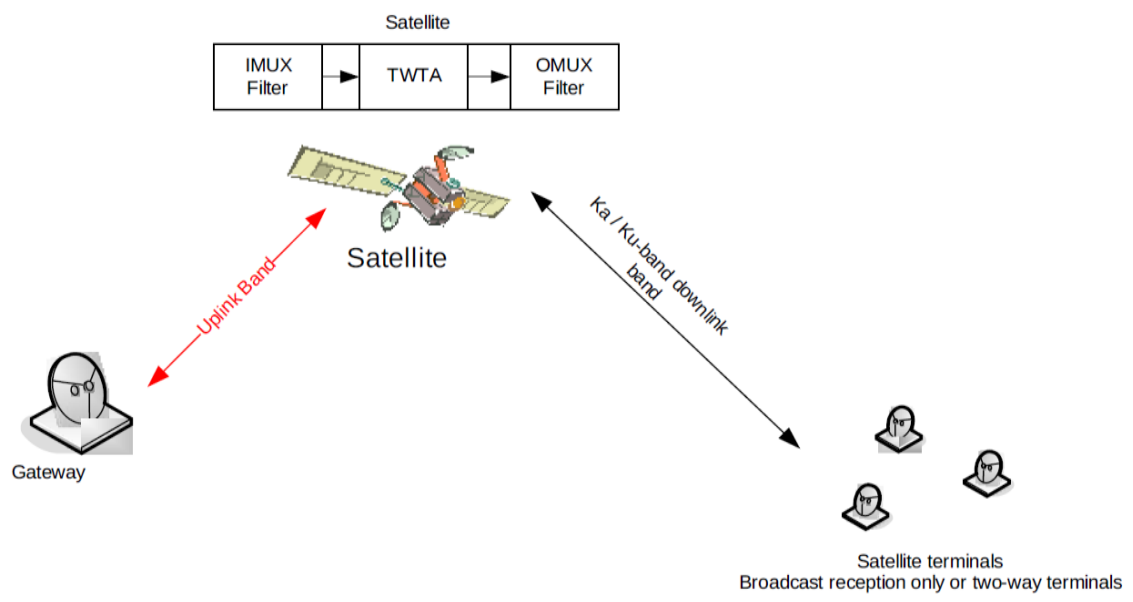

Figure 1 Satellite System Scenario

\section{B. Non-linear Satellite Channel}

The overall channel model is shown in Figure 2 for the case of three carriers where we can identify the multicarrier predistortion block at the gateway and the single carrier equalization blocks at the terminal receivers. The uplinked signal from the gateway is channelized and amplified in the satellite transponder. IMUX and OMUX filter responses are depicted in Figure 2 for the case of a $36 \mathrm{MHz}$ transponder bandwidth.

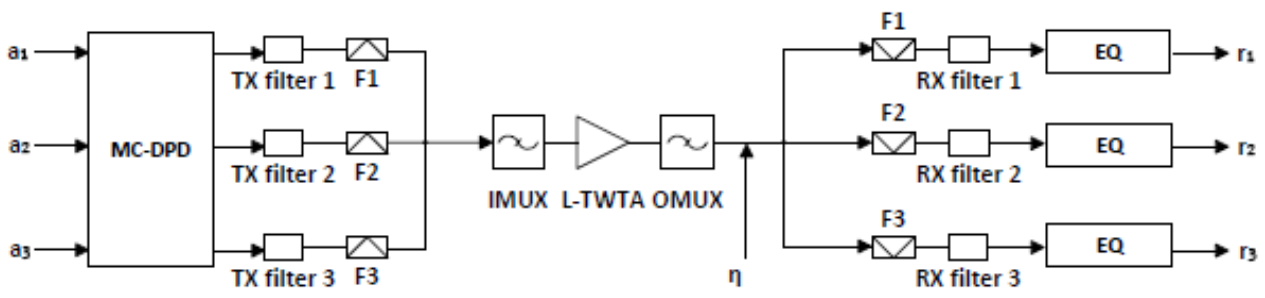

Figure 2 Channel Model 
On-board HPAs are implemented with TWTAs that are intrinsically non-linear, especially when operated in their high efficiency region. However, partial linearization of the TWTA amplifier can be achieved on-board by means of specific RF technology resulting in the Linearized-TWTA (L-TWTA). Further, the TWTAs used in Ku-band can be assumed to have a transfer characteristic largely independent of the frequency. Such memoryless amplifier functions are characterized by the AM/AM and AM/ PM curves. These curves are depicted in Figure 4 for the considered TWTA and L-TWTA, respectively.
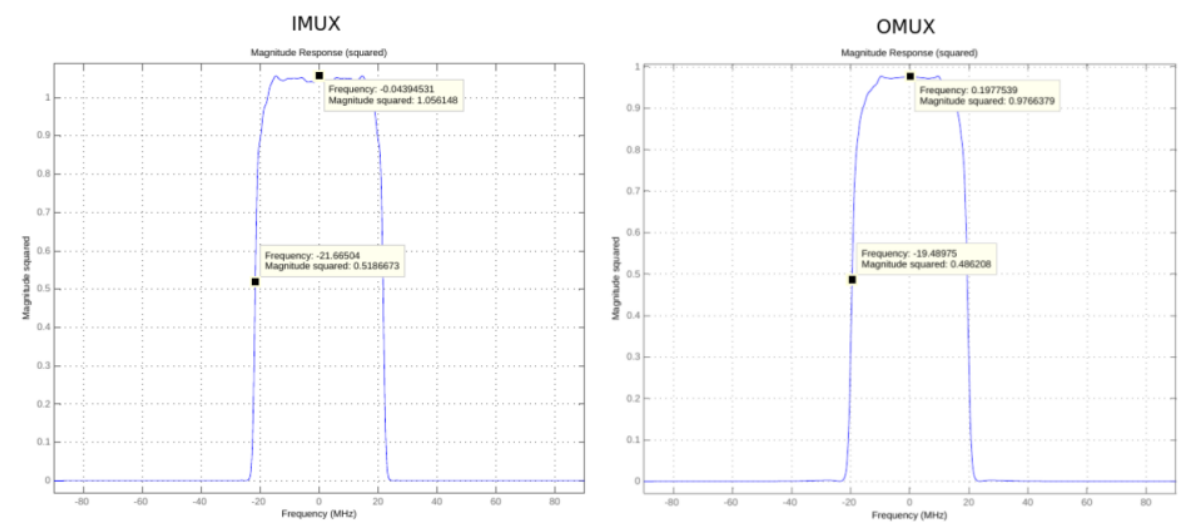

Figure 3 IMUX and OMUX characteristics

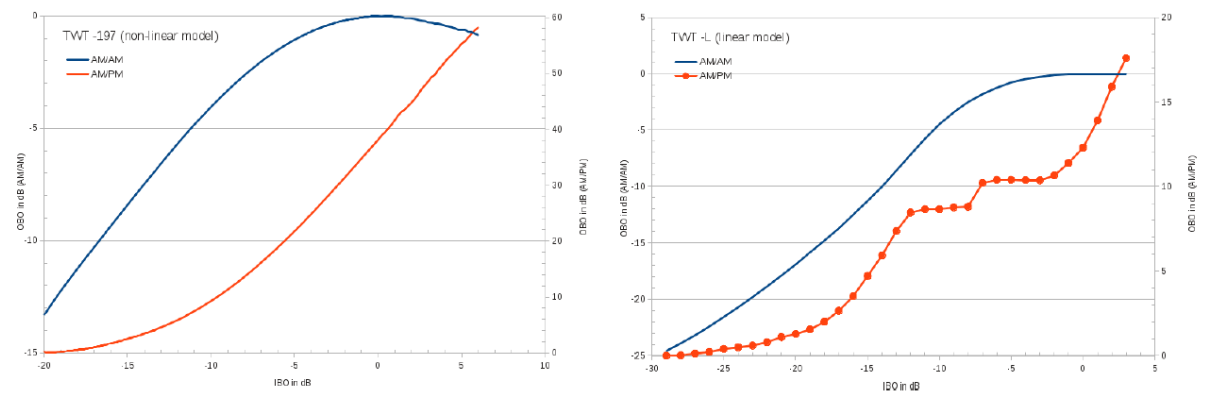

Figure 4 AM/AM AM/PM Characteristic of LUT based TWT 197 and LTWT

The channel being non-linear with memory, we not only have constellation warping effects but significant interference. Inter-symbol Interference is generated by the memory effect combined with the non-linear characteristic of the amplifier. However, in our scenario, the dominant interference effects are the non-linear ACI excited by the intermodulation products generated by the multicarrier joint amplification as detailed in Ref. 1 . These are depicted in Figure 5 where the noiseless scatter plot of the received 16 APSK symbols on the central carrier of a three carrier system is presented (on the right). The corresponding effect for the single carrier channel is also shown (on the left) to highlight the dominant effect of ACI. 

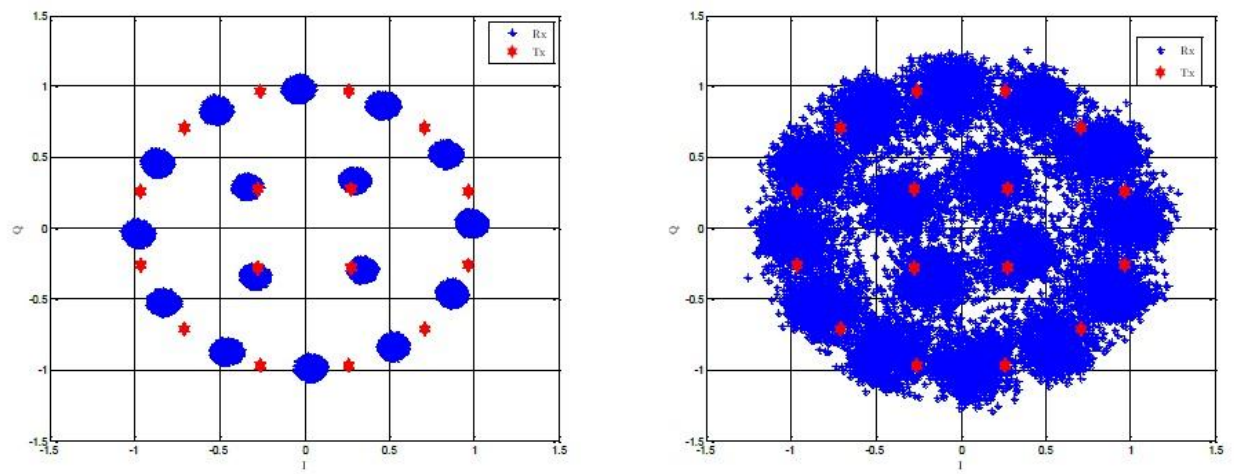

Figure 5 Noiseless Scatter Plots depicting received symbols and interference

\section{Enhancement techniques DPD and EQ}

\section{Digital Pre-Distortion (DPD)}

Digital pre-distortion operates in the gateway (transmitter side) seeking to invert the channel transfer function, such that the cascade of DPD and the channel is as linear as possible.

\section{Signal and Data DPD}

Data DPD operates at symbol level, prior to the pulse-shaping filter, as depicted in Figure 6. This scheme has the advantage of not increasing the bandwidth of the predistorted signal compared to a signal DPD scheme. A data predistorter operates on the baseband data symbols. It modifies the transmitted constellation in such a way that, after subsequent GW and transponder processing, the received constellation at the detector would match the desired signal constellation on an average.

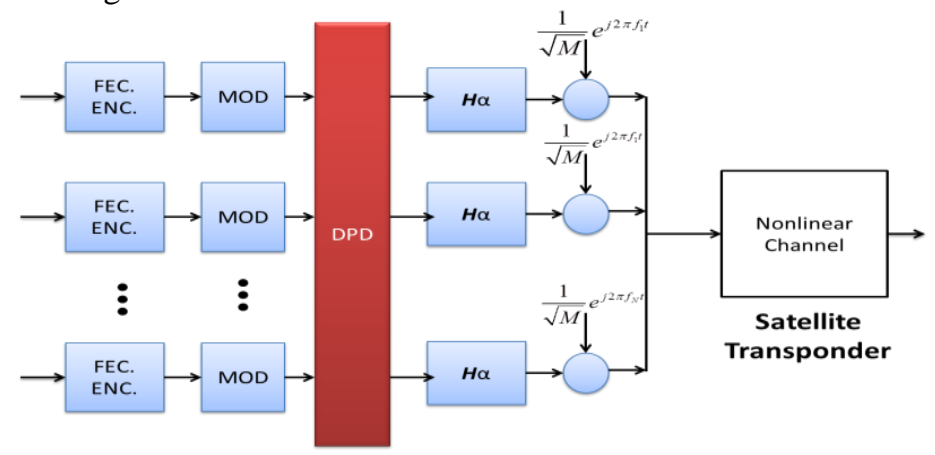

Figure 6: Multi-carrier scheme with Data Digital Pre-Distortion (DPD)

A signal (or waveform) pre-distorter generates a signal that compensates for the linear and nonlinear distortions introduced by the RF module. Signal predistortion has the disadvantage that generates signals with higher bandwidths. However, it is usually more precise since it operates in a domain where the distortion is better described.

\section{Candidate techniques for DPD}

From the investigation carried out in Ref. 1, we selected the characteristics of the predistortion function: 
- Data predistortion is preferred over signal predistortion. In this case the signal bandwidth is the same as the predistortion bandwidth; no up-sampling is made and hence the main advantage of signal predistortion is not present. Furthermore, data predistortion is easier to implement.

- Indirect learning is easier to implement than direct learning. Indirect learning is easily made adaptive and the requirements on the feedback are not that hard. Direct learning is also of no use when a tabulated channel is used as is often the case here.

- Model based predistortion is preferred. A look-up-table would require very large number of coefficients and would also be difficult to make adaptive (which is discussed in more detail below)

Simulations reveal that in a multicarrier scenario the outer carriers are affected by memory effects (ISI). The carriers located close to the center of the band are practically unaffected by memory effects; however they suffer from heavier distortion caused by the outer carriers.

The nonlinear cross talk effects, manifested in the form of ACI, are significantly larger than memory effects and is in fact the dominating distortion.

Three different candidates to perform the DPD in a multicarrier scenario were selected from the literature and further studied: look-up tables ${ }^{2}$ (LUT), memory polynomials ${ }^{4}$ (MP) and Volterra basis $^{3}$ (V).

\section{Memory polynomial:}

- The most attractive predistortion algorithm (small number of coefficients, includes memory and nonlinear effects; linear optimization can be applied).

- Identification using least square method (pseudo inverse of matrix) is a standard and robust process.

- The nonlinear order and memory depth must be optimized for each scenario [number of carriers, (IMUX-TWTA-OMUX)].

- Excellent adaptivity properties when used in combination with structures like Kalman filter.

\section{Volterra basis:}

- Theoretically preferred, since it is valid for any non-linear dynamic system.

- Slow convergence, high number of parameters lead to numerical problems

- Comparable performance to memory polynomials.

- Adaptivity possible with Kalman filters but with reduced convergence speed.

\section{Look-up Table (LUT):}

- LUT is not recommended for DPD in a multicarrier scenario, due to the very large size of these tables required.

- Including all ACI and ISI, the size of the LUT is $N M^{L N}$, where $\mathrm{M}$ is number of symbols, $\mathrm{L}$ is the memory depth, $\mathrm{N}$ number of carriers. (e.g.: $\mathrm{M}=32, \mathrm{~L}=3, \mathrm{~N}=5$ gives a LUT-size of $1.8889 \mathrm{e}+023$ ). Reduction may be possible due to symmetries of the respective constellation diagrams. An example of the reduction for a single carrier is $3 / 16$, i.e. not significant.

- Include all ACI and ISI only for outmost channels: LUT-size $=2 M^{L+N-1}+(\mathrm{N}-2) \mathrm{M}^{\mathrm{N}}$. (e.g. $\mathrm{M}=32$, $\mathrm{L}=3, \mathrm{~N}=5$ gives a LUT-size of $6.8820 \mathrm{e}+010$, i.e. also not feasible).

- If different modulations are used for different carriers, each combination will require a new LUT of the size as given above.

- Adaptivity would be difficult and slow since so many coefficients have to be updated.

Table 1 summarizes the evaluation performed for these three DPD candidates. 
Table 1: Evaluation of three candidate DPD structures in a multicarrier scenario

\begin{tabular}{|c|c|c|c|c|}
\hline DPD Method & Performance & Complexity & Adaptivity & Comments \\
\hline LUT & $?$ & Very High & Bad & $\begin{array}{c}\text { Performance and adaptivity difficult } \\
\text { to evaluate due to the complexity. }\end{array}$ \\
\hline $\begin{array}{c}\text { Memory } \\
\text { Polynomial (MP) }\end{array}$ & Good & Low & Good & Most attractive \\
\hline Volterra (V) & Medium & High & Medium & $\begin{array}{c}\text { Performance and adaptivity reduced } \\
\text { compared to MP to evaluate due to } \\
\text { the complexity. }\end{array}$ \\
\hline
\end{tabular}

\section{DPD model}

The DPD model implemented is a memory polynomial described by sum of linear terms and third order and fifth order terms.

Let's assume $x_{t}(n)$ and $y_{t}(n)$ are the $n$-th sampled instants of the t-th carrier (denoted in its baseband form) for both the input and output of the predistorter function, then, the relationship between $y_{t}(n)$ and $x_{t}(n)$ is described by the following equations:

$$
\mathrm{y}_{\mathrm{t}}(\mathrm{n})=\mathrm{g}_{\mathrm{t}}^{(1)}+\mathrm{g}_{\mathrm{t}}{ }^{(3)}+\mathrm{g}_{\mathrm{t}}{ }^{(5)}
$$

Linear terms:

$$
\mathrm{g}_{\mathrm{t}}^{(1)}=\sum_{\mathrm{m}=0}^{\mathrm{M}_{1}} \mathbf{h}_{\mathrm{i}}^{(1)}(\mathrm{n}) \mathrm{x}_{\mathrm{i}}\left(\mathrm{n}-\mathrm{m}_{1}\right) \quad \mathrm{i}=1,2 \ldots \mathrm{P}
$$

Third order terms:

$$
g_{t}^{(3)}=\sum_{11=0}^{\mathrm{M}_{3}} \sum_{12=11}^{\mathrm{M}_{3}} \sum_{13=0}^{\mathrm{M}_{3}} \mathbf{h}_{\mathrm{i}, \mathrm{j}, \mathrm{k}}^{(3)}(\mathrm{n}) \mathrm{x}_{\mathrm{i}}(\mathrm{n}-11) \mathrm{x}_{\mathrm{j}}(\mathrm{n}-12) \mathrm{x}_{\mathrm{k}}{ }^{*}(\mathrm{n}-13) \quad \mathrm{i}, \mathrm{j}, \mathrm{k}=1,2 \ldots \mathrm{P}
$$

Fifth order terms:

$$
\begin{aligned}
& g_{t}{ }^{(5)}=\sum_{11=0}^{M_{5}} \sum_{12=11}^{M_{5}} \sum_{13=12}^{M_{5}} \sum_{13=0}^{M_{5}} \sum_{14=13}^{M_{5}} h_{i, j, k, 1, m}^{(5)}(n) x_{i}(n-11) x_{j}(n-12) x_{k}(n-13) x_{1}{ }^{*}(n-14) x_{m}{ }^{*}(n-15) \\
& " i, j, k, 1, m=1,2 \ldots P
\end{aligned}
$$

The sub-indices of the carriers permute for all possible combinations, using $\mathrm{i}, \mathrm{j}, \mathrm{k}, \mathrm{l}, \mathrm{m}=1,2, \ldots \mathrm{P}$. Being $\mathrm{P}$ the total number of carriers.

The variables $M_{1}, M_{3}, M_{5}$ denote the memory of the linear, third and fifth order terms. Simulations have shown that the impact of memory terms decrease as the nonlinear order increases; where the impact of the memory in for third and fifth order terms is small compare to the memory effects encountered in the linear term. 
Notice that the summations avoid repetitive terms this is included in the DPD to ensure numerical robustness to the identification process.

The basis (summands) of the DPD model is reduced by ignoring the terms which contribution is out of band. Thus, the terms which contribution appears in-band are kept in the model as in Ref. 10.

\section{Identification}

Digital pre-distortion requires knowledge of the channel characteristics. Towards this, it is assumed the existence of dedicated receiver terminals, which function is to provide output data from the channel which will be used to find estimates of the predistorter function.

Direct and indirect learning - Adaptivity

DPD co-efficients are identified using either the direct or the indirect learning method, respectively. The methods are illustrated in. Using the indirect learning method, the coefficients in the algorithm for the inverse of the nonlinear system are identified in a first step and in a second step copied to the predistorter (Figure 7).

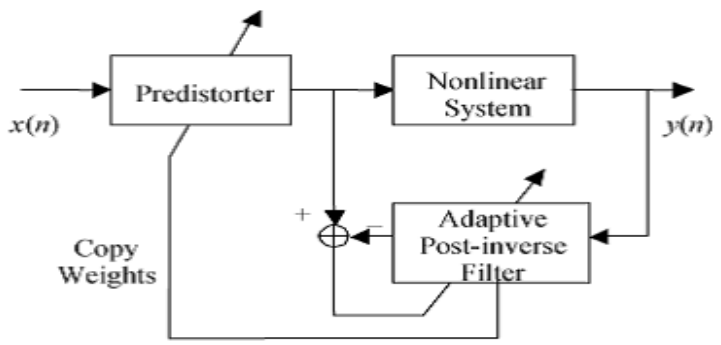

(a)

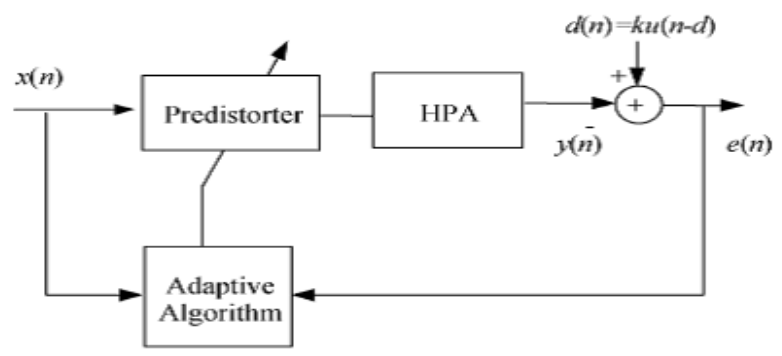

(b)

Figure 7 DPD Identification

The indirect learning method is based on the fundamental " $p$ th order theorem" that states the post inverse and pre inverse of a nonlinear dynamic system are identical and that the nonlinear order $(p)$ of the system's inverse is the same as the nonlinear order of the system itself. The indirect learning method has the advantage that it is easy to implement. It does not require any real time feedback. It has been suggested that the indirect learning method is more sensitive to noise; this problem can be overcome by making it adaptive as described below.

In direct learning, the coefficients of the predistorter are updated directly based on its input and reference error. The direct learning method has the advantage of being adaptive, but its implementation is more difficult.

The most straightforward identification of the coefficients in the indirect learning method with Volterra or memory polynomial is through the use of least squares method. Here, all coefficients are obtained from the pseudo-inverse 
of the system's transfer matrix. The disadvantage is that all coefficients are updated simultaneously and that there is no sliding memory.

\section{B. Equalization}

While multicarrier digital predistortion is implemented at the transmitter side, in reality, it cannot fully compensate the distortion effects of the channel. Digital predistortion implemented in the form of a memory polynomial function is well suited to compensate for the effects of IMUX and HPA, while the remaining distortion introduced by the OMUX filter can be effectively addressed by single carrier linear equalization at the receiver side. Moreover, equalization at the receiver can be easily made adaptive capable to track fast channel variations with the aid of dedicated training symbols.

\section{Identification}

For a given order and memory, estimation of the kernel co-effcients can be formulated as a Linear Least Squares problem In this paper, a standard Recursive Least Squares implementation is considered to reduce the complexity and to be able to track channel changes ${ }^{8,9}$. In all equalization cases, Recoursive Least Squares (RLS) technique is employed to iteratively adapt the kernel coefficients to channel changes according to the following set of equations:

$$
\begin{gathered}
\mathrm{u}(\mathrm{i})=\left[\begin{array}{c}
\mathrm{x}(\mathrm{i}) \\
\vdots \\
x(i-N)
\end{array}\right] \\
\mathrm{e}(\mathrm{i})=\mathrm{d}(\mathrm{i})-\mathrm{u}(\mathrm{i})^{\mathrm{T}} \mathrm{h}(\mathrm{i}-1) \\
\mathrm{g}(\mathrm{i})=\frac{\mathrm{P}(\mathrm{i}-1) \mathrm{u}(\mathrm{i})^{*}}{\gamma+\mathrm{u}(\mathrm{i})^{\mathrm{T}} \mathrm{P}(\mathrm{i}-1) \mathrm{u}(\mathrm{i})^{*}} \\
\mathrm{P}(\mathrm{i})=\gamma^{-1} \mathrm{P}(\mathrm{i}-1)-\mathrm{g}(\mathrm{i}) \mathrm{u}(\mathrm{i})^{\mathrm{T}} \gamma^{-1} \mathrm{P}(\mathrm{i}-1) \\
\mathrm{h}(\mathrm{i})=\mathrm{h}(\mathrm{i}-1)+\mathrm{e}(\mathrm{i}) \mathrm{g}(\mathrm{i})
\end{gathered}
$$

where $\mathrm{u}(\mathrm{i})$ is the vector of all the linear terms included in the equalization function (form and number of terms depend on the type of model, degree and memory depth), $h(i)=\left[h_{1}^{i}(0), \cdots h_{1}^{i}(K-1)\right]^{T}$, is the vector consisting of the kernel coefficients during the $\mathrm{i}^{\text {th }}$ instance, $\mathrm{d}(\mathrm{i})$ is the desired symbol and $\gamma$ the forgetting factor.

Each frame is assumed to have a dedicated code_seg1 of 90 training symbols in the target modulation. This allows supporting Adaptive Code Modulation operation mode foreseen by the standard, as well as estimating the drift in channel parameters. From simulation results the forgetting factor has been set to 0.995 .

\section{Simualtion Results}

Extensive simulations have been performed to verify the validity of the described techniques. The performance and sensitivity of the key channel parameters is investigated with respect to the total degradation defined as:

$$
\mathrm{TD}=\left.\frac{\mathrm{E}_{\mathrm{b}}}{\mathrm{N}_{0}}\right|_{\mathrm{NL}}-\left.\frac{\mathrm{E}_{\mathrm{b}}}{\mathrm{N}_{0}}\right|_{\text {Ideal }}+\text { OBO. }
$$

The term $\left.\frac{E_{b}}{N_{0}}\right|_{N L}-\left.\frac{E_{b}}{N_{0}}\right|_{\text {Ideal }}$ reflects the loss in SNR of a practical HPA compared to ideal HPA for achieving the same $\mathrm{BER}$ at the same OBO levels. This term is penalized by OBO to reflect on the loss in power efficiency with high OBO. As OBO increases, the practical HPA is pushed more and more into the linear region and $\left.\frac{\mathrm{E}_{b}}{\mathrm{~N}_{0}}\right|_{\mathrm{NL}}-\left.\frac{\mathrm{E}_{\mathrm{b}}}{\mathrm{N}_{0}}\right|_{\text {Ideal }}$ reduces. Thus one could see a trade-off between the two components and an optimum OBO minimizing the TD is usually seen. As a measure of bandwidth usage efficency we define: 


$$
y=\frac{\operatorname{Rate}(1+\alpha)}{\Delta f}
$$

where $\Delta f$ is the carrier separation (we assume equally spaced carriers) and $\alpha$ the roll off.

\section{A. Simulation Parameters}

In this work, we study the performance variation of a multicarrier non-linear satellite channel to different channel parameters with DPD and Equalization in place to improve spectral and power efficiency. OBO provides a measure of power efficiency while total degradation is related affects the overall link budget. Spectral efficiency is instead regulated by many transmission parameters:

- Code Rate

- Modulation order

- Total occupied bandwidth with respect to available transponder bandwidth

- Carrier Spacing/ Number of carriers

○ Roll Off

Moreover two different amplifier models are considered in this work:

- TWTA : LUT model extracted from Figure 4 left

- $\quad$ Linearized TWTA: LUT model extracted from Figure 4 right

In the sequel we directly refer to the followings predistortion and equalization techniques:

- EQ1: Linear equalization with 3 memory taps.

- EQ21: Linear equalization with 21 memory taps.

- DPD53: Multicarrier digital predistortion with polynomial degree 3 and memory depth 5.

- DPD55: Multicarrier digital predistortion with polynomial degree 5 and memory depth 5.

In the following, we first evaluate the channel performance sensitivity, in terms of total degradation, for various configurations. Secondly we evaluate the gains of predistortion and equalization for some significant scenarios.

Shown total degradation results are reduced based on natural channel symmetric characteristics: for two carriers only one carrier is shown; for a three carrier scenario we show only the central and external carrier performance results; in four carrier scenario we distinguish internal and external carriers only.

\section{B. Channel Results}

\section{- LTWTA/TWTA}

In Figure 8 we have the total degradation for a dual carrier channel configuration when the on-board amplifier model changes from TWT to L-TWTA.It is clear that the use of a L-TWTA provides for improvement both in the power efficiency (OBO improvement of $1 \mathrm{~dB}$ ) as well as the SNR gain (of about $0.5 \mathrm{~dB}$ ) 


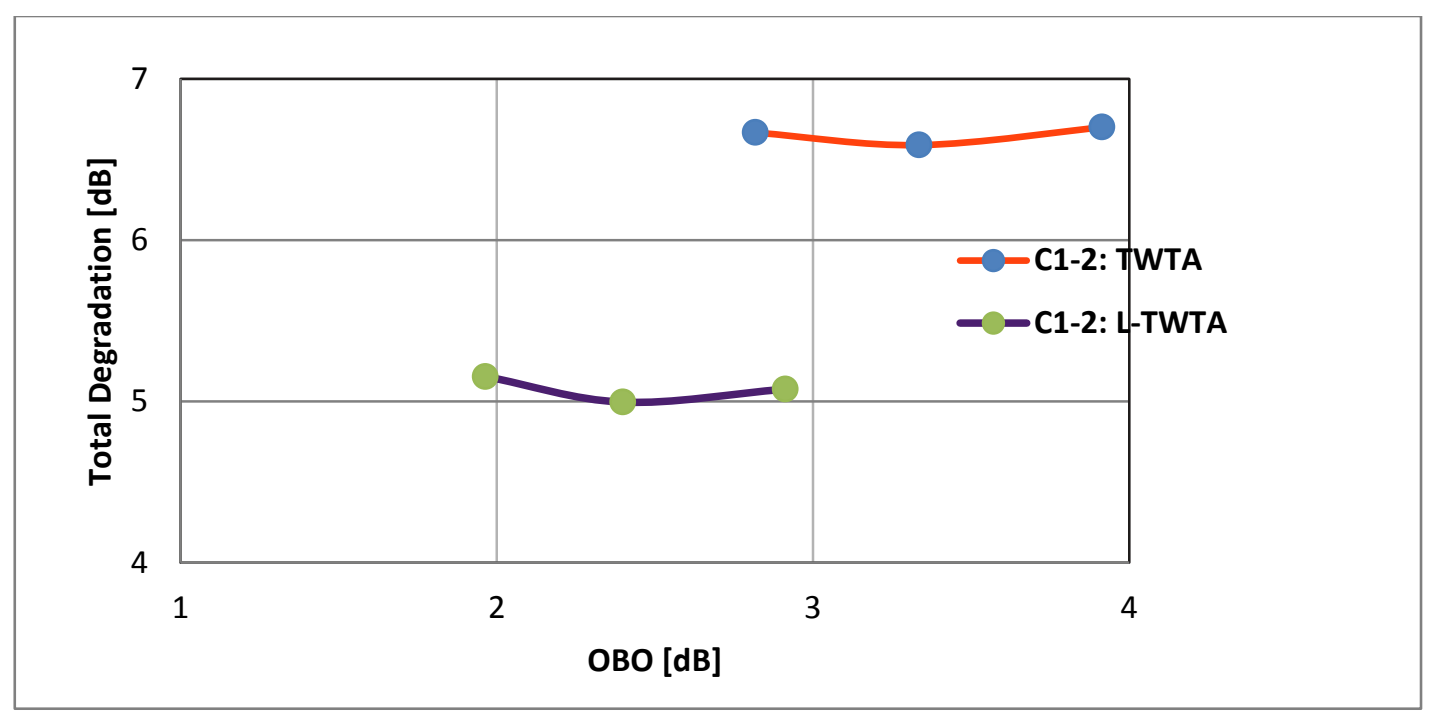

Figure 8 Dual Carrier Channel with TWTA vs L-TWTA with $\alpha=.2 \gamma=1.15$ 16 APSK 13.85 MBaud, Bandwidth=28.6 MHz

\section{- Filters Effects: MUX on/off}

Total degradation curves in Figure 9 evaluate the effects of IMUX/OMUX filtering. Figure 9 also shows how close is in this case the performance of the strongly non linear channel but without MUX filters, to the configuration where the Linearized TWT is employed.

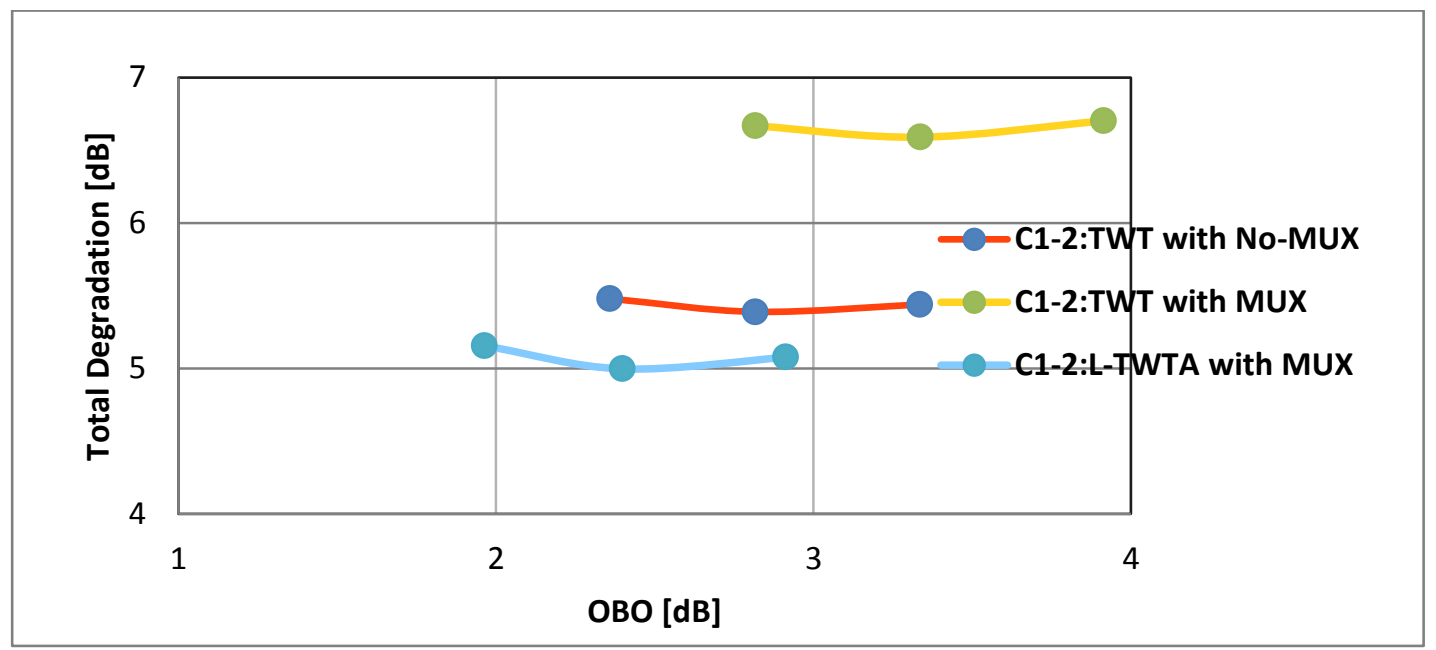

Figure 9 Dual Carrier Channel with/without MUX filters with $\gamma=1.15 \alpha=.2$ dual carrier 16APSK 13.85 MBaud, Bandwidth $=28.6 \mathrm{MHz}$

\section{- Roll Off /Carrier Spacing}

In this section we see how the total degradation varies with respect to spectral efficiency. Spectral efficiency is determined here by roll off and $\gamma$ for a dual carriers channel. 


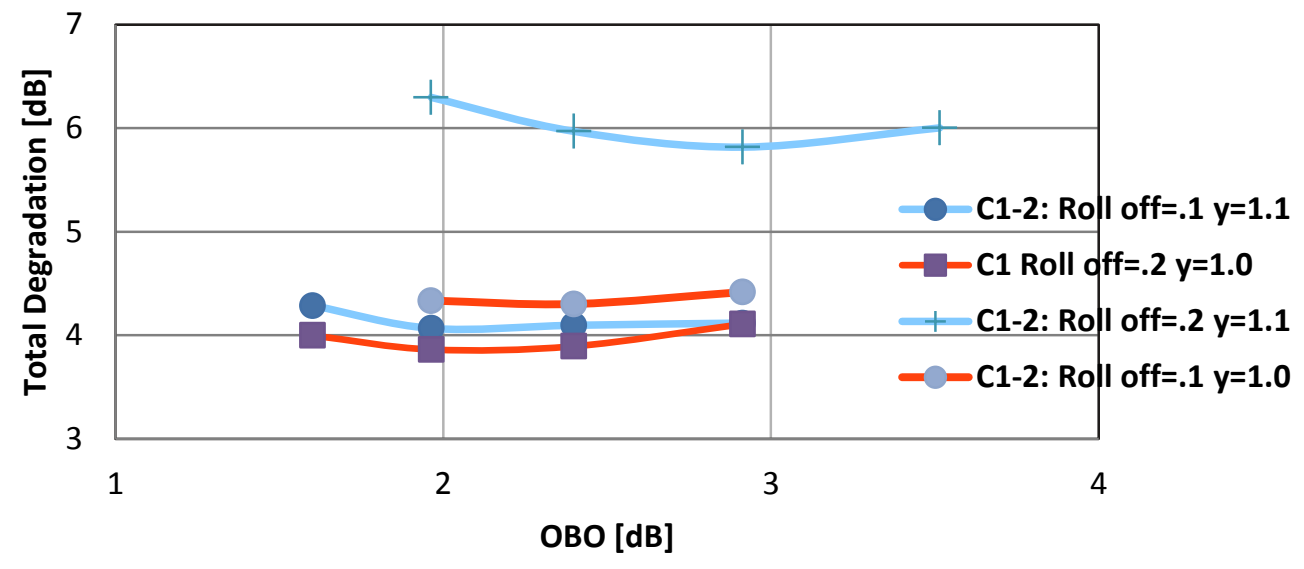

Figure 10 Effect of roll-off and Igamma on TD performance in a Dual Carrier channel with 16.36 MBaud, 16 APSK, L-TWTA

In Figure 10, we notice how roll off reduction produces a loss in performance when the carrier the carrier separated $(\gamma=1)$. This is due to the fact that lower roll-off increases the peak to average power ratio causing enhanced degradation. On the other hand, when carriers overlap, roll off reduction drastically improves performance. This arises from the fact that degradation due to overlap is dominant and offsets any change due to roll-off variations.

\section{- Coderate}

In Figure 11, we illustrate how coderate affects TD. Figure 11 depicts that a stronger code rate tolerates higher interference. This also provides an improvement of power efficency reducing the minimum OBO. The explanation is that the distortion due to mixing products from one carrier is uncorrelated with the signal at the other carrier; the decoder that is designed for white noise will, hence, have an impact also on the mixing products.

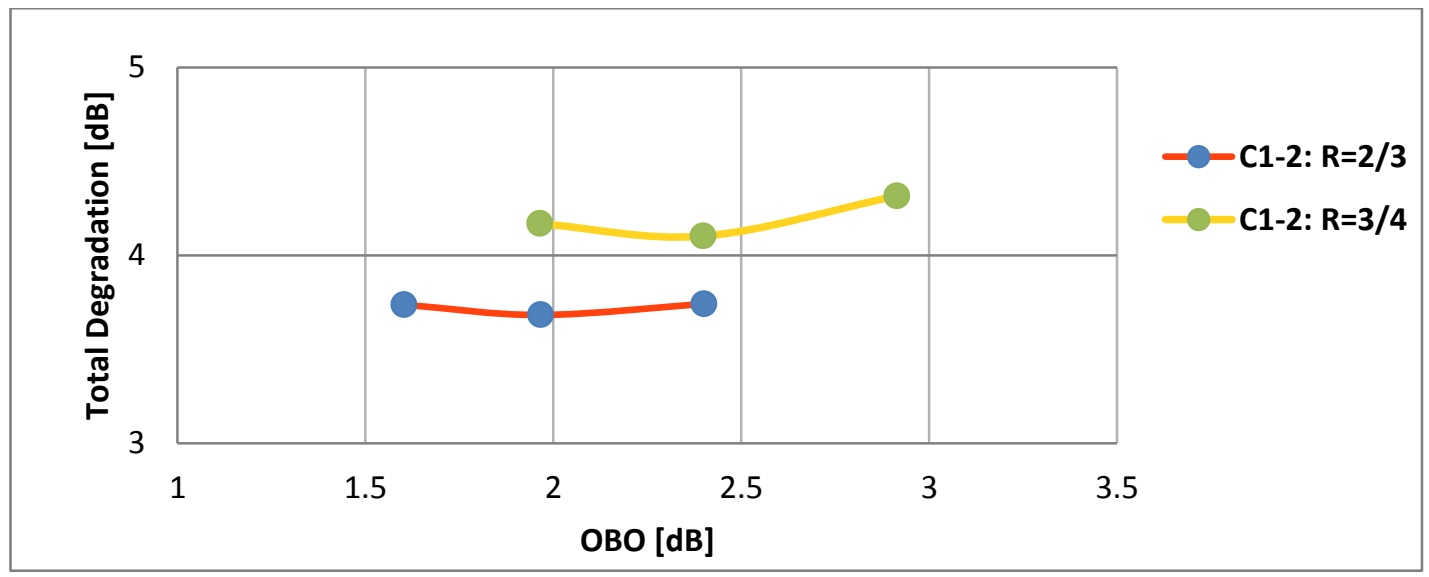

Figure 11 Effect of code rate on TD performance in a dual carrier scenario with 15 Mbaud,16 APSK, $\alpha=0.2, \gamma=1.1$, L-TWTA, Bandwidth $=31.6 \mathrm{MHz}$ 


\section{Digital Pre-Distortion with Equalization Results}

In this section, we provide results on multicarrier digital predistortion for different channel configurations. Figure 12 compares a dual carrier satellite channel where a significant overlapping factor is applied $(\gamma>1)$. Two predistortion settings are here considered: DPD53 and DPD55. In both cases EQ1 is performed at receivers. As a bench mark for comparison we have results when only EQ1 is applied. Both predistortion configurations outperform EQ1. From Figure 12 we can also conclude that increasing the polynomial degree from three to five (DPD53 and DPD55) tends to improve the performance.

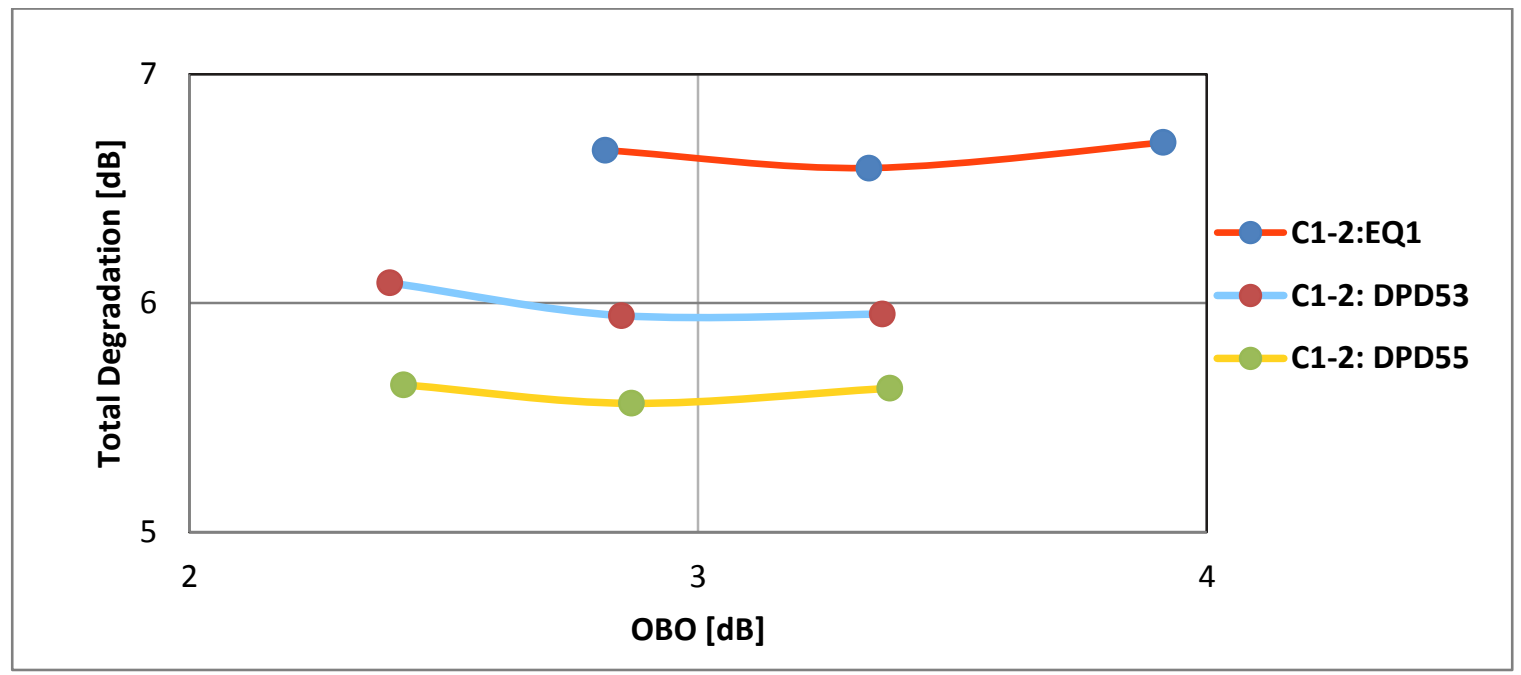

Figure 12 Dual Carrier channel with different predistortion configurations, 13.85 Mbaud , 16 APSK, $\alpha=0.2$, $\gamma=1.15$, TWTA, Bandwidth $=28.6 \mathrm{MHz}$

Predistortion performance and relative gains strongly depend on the channel non-linear characteristic. In Figure 13 we observe how predistortion performance is affected by the choice of the on-board HPA. As expected, the relative gain of DPD over EQ1 is greater when we choose the non-linearized amplifier (TWTA). However absolute performance is always better with L-TWTA.

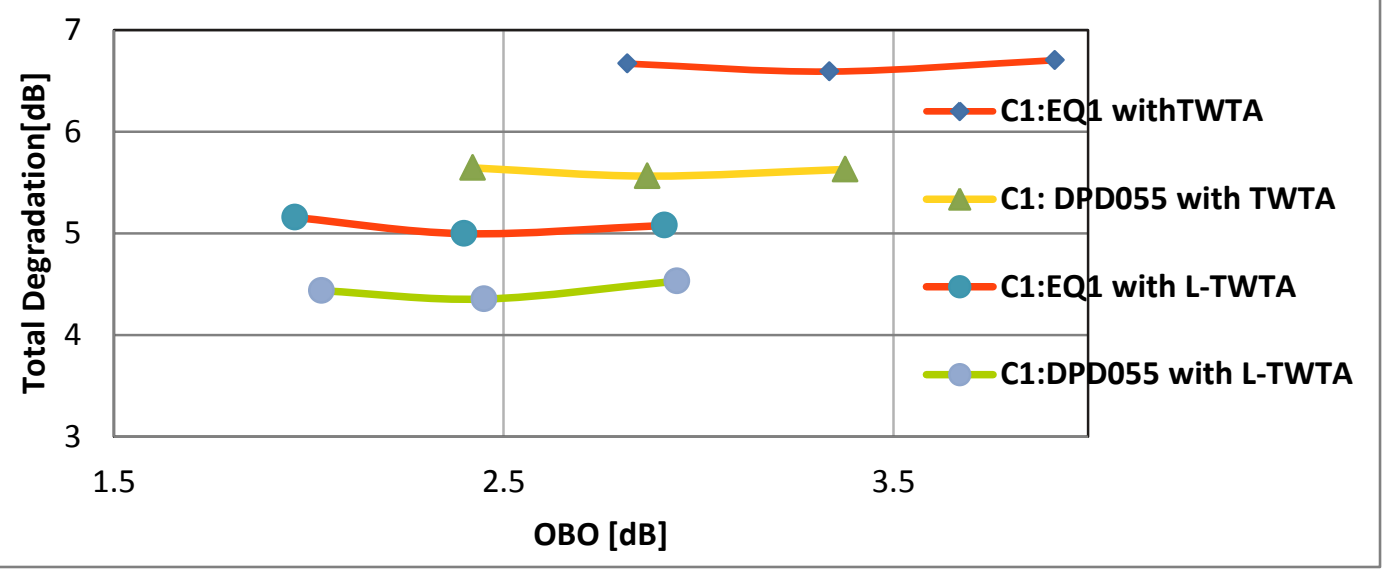

Figure 13 Dual Carrier channel predistortion performance for TWTA and L-TWTA, 13.85 Mbaud,16 APSK, $\alpha=0.2, \gamma=1.15$, Bandwidth $=28.6 \mathrm{MHz}$

As already seen in the general channel results, the choice of code rate affects significantly total degradation performance. Relative improvement is obtained at the cost of spectral efficiency reduction. In Figure 14 we compare predistortion performance with respect to the code rate. 


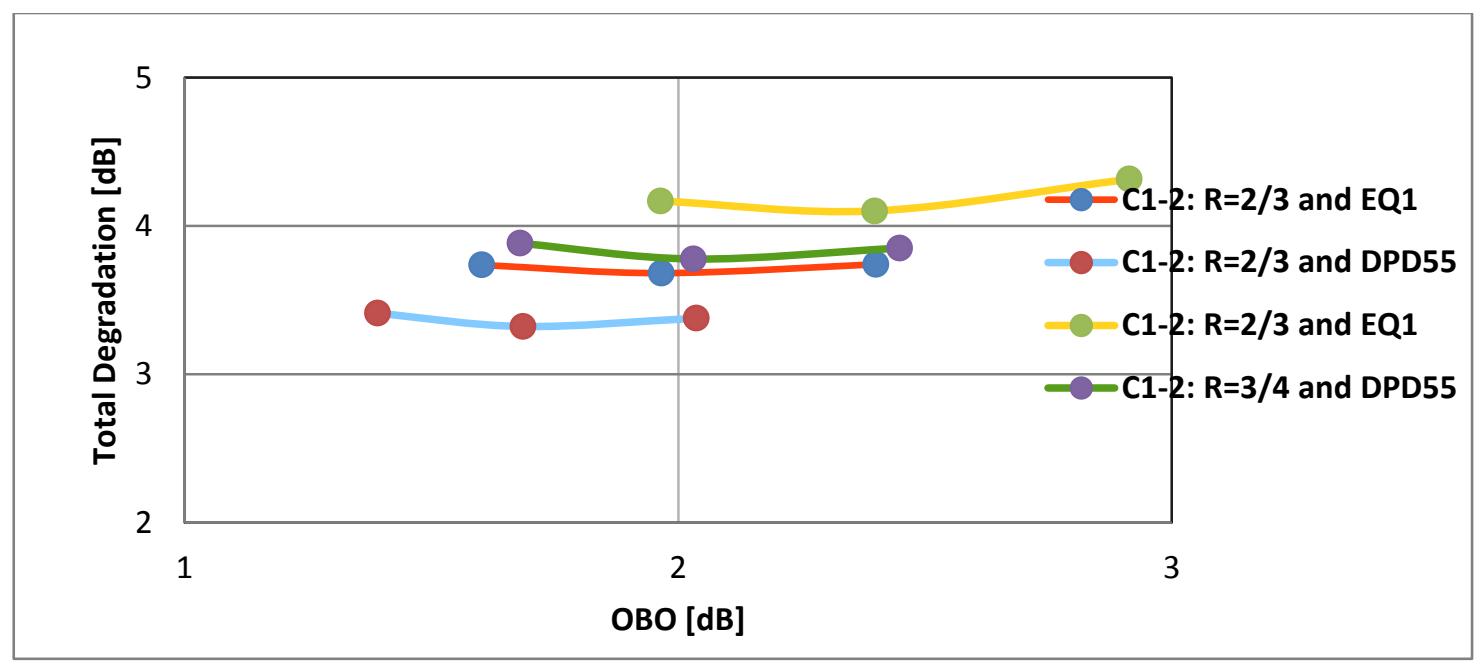

Figure 14 Dual Carrier channel predistortion performance for Code Rate 2/3 and 3/4, 15 Mbaud,16 APSK, $\alpha=0.2, \gamma=1.1$, L-TWTA, Bandwidth $=31.6 \mathrm{MHz}$

In Figure 14, comparing for each code rate predistortion performance with respect to its EQ1 as benchmark curve, it can be concluded that relative gains are basically invariant.

In Figure 15, we have results for a three carrier channel. As expected, performance of the central carrier is in general worse than the external ones. This is a consequence of the prominent adjacent channel interference on the internal channel. Digital predistortion is shown to be effective in reducing the TD, in the external, as well internal channels by about $1 \mathrm{~dB}$.

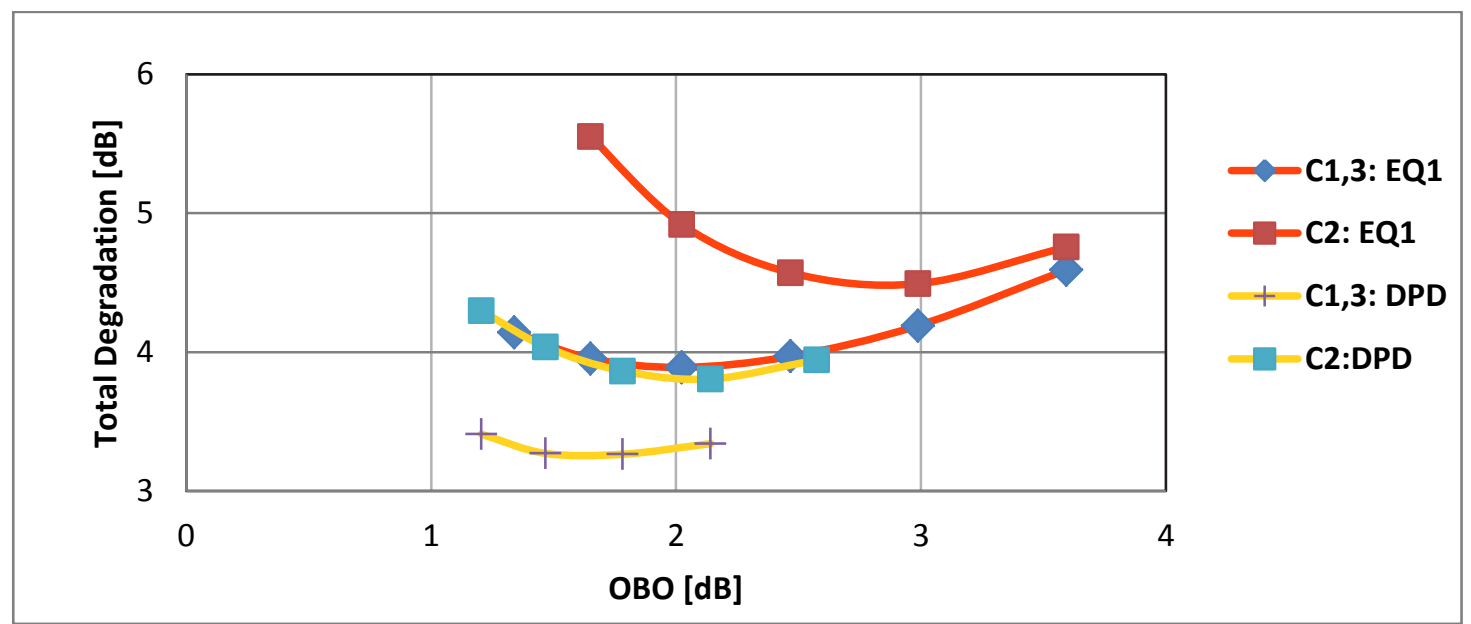

Figure 15 Performance of DPD in a Three Carrier Satellite channel: 10Mbaud, $\gamma=0.91,16-16-16$ APSK, Bandwidth $=33.9 \mathrm{MHz}$

A four carrier experiment is reported in Figure 16. Inner and outer carriers, in pairs, have similar performance. In this very tight scenario where the number of intermodulation products is very high, predistortion is even more effective providing very significant gain reducing the total degradation of about $1 / 1.5 \mathrm{~dB}$ and improving power efficiency (optimum OBO) of about $2 \mathrm{~dB}$. 


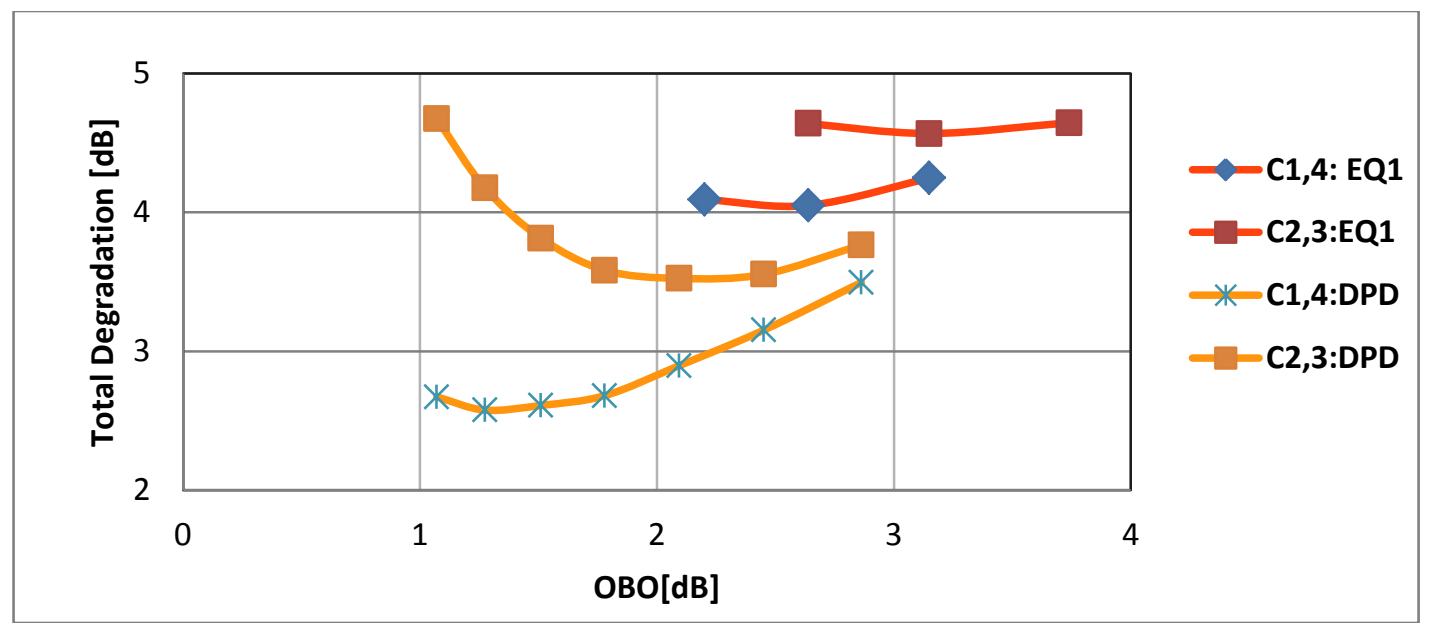

Figure 16 Performance of DPD in a four Carrier Satellite Channel: 6.9 Mbaud, $\gamma=.93,32-16-16-32$ APSK, Bandwidth $=30.5 \mathrm{MHz}$

\section{Equalization Only with high memory depth}

In this section we introduce as interference mitigation technique long memory single carrier linear equalization at the receivers. For this evaluation we do not apply any joint predistortion at the transmitting gateway. In Figure 17 we can evaluate the performance gain provided by 21 taps linear equalization EQ21 with respect to thee taps linear equalization EQ1 in a dual carrier channnel also depending on the parameter $\gamma$.

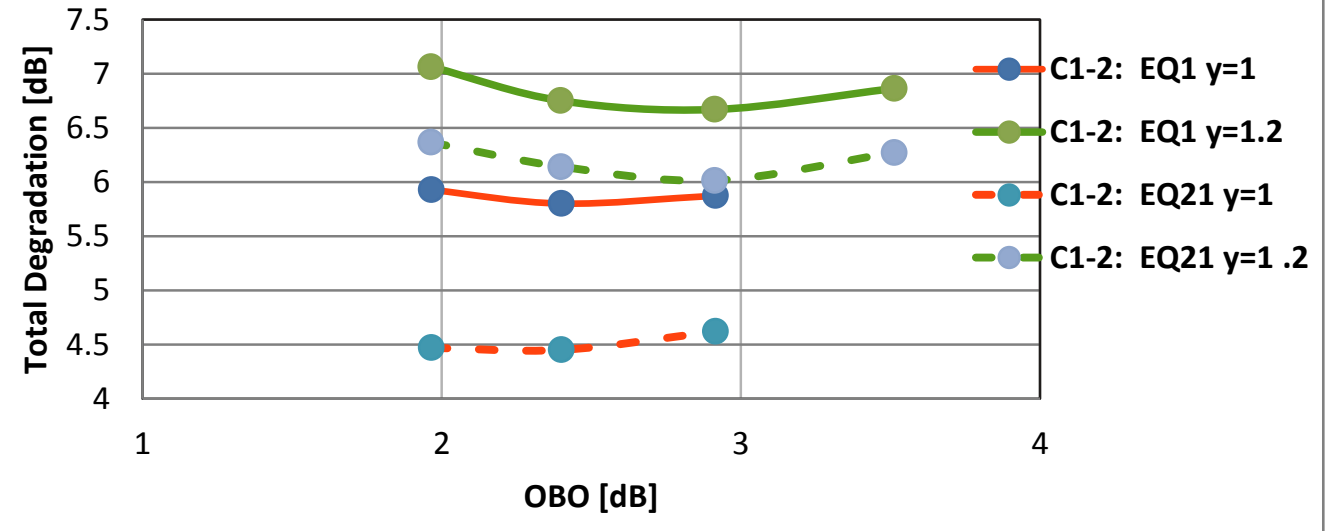

Figure 17 Dual Carrier channel with linear equalization having long memory: 18 Mbaud, 16 APSK, $\alpha=0.2$.

From results shown in Figure 17, we can conclude that EQ21 provides a significant gain even in case of perfect carrier separation. Moreover, EQ21 becomes more effective as the carrier spacing decreases $(\gamma>1)$ with a resulting increase in linear ACI.

\section{Conclusions}

The paper investigated the contributions of various channel parameters to the degradation and analysed the performance of non-linear mitigation techniques for multiple carrier satellite channel with respect to these key channel parameters. Channel performance shown to be sensitive to many channel parameters such as overlapping, 
Roll Off, code rate etc. Digital predistortion reduces the amount of interference at the receiver, while increasing the HPA power efficiency by moving the TD minimum closer to the amplifier high efficiency region. Results show that DPD provides better relative gain when the amplifier is strongly non-linear. Moreover, TD improvements become more significant when the number of carriers increases showing the advantage of joint processing. Single carrier equalization provides notable gains when long memory is employed especially in the scenario when high spectral overlap.

In conclusion the considered on-ground processing techniques provide significant Total Degradation gain at low OBO region $(2-3 \mathrm{~dB})$.

\section{Acknowledgments}

The authors would like to thank the European Space Agency (ESA) for their support through the ARTES 5.1 project "On Ground Multicarrier Digital Equalization/Predistortion Techniques for Single or Multi Gateway Applications" (APEXX) - ESA Contract No.: 4000105192/12/NL/AD. Moreover, the authors would like to thank particularly Mr. Nicolas Girault from ESA/ESTEC (TIA).

\section{References}

\footnotetext{
${ }^{1}$ R. Piazza, E. Zenteno, and e. al, "Multicarrier digital predistortion/equalization techniques for non-linear satellite channels,"in Proc. 30th AIAA Intern.Commun. Satellite Syst. Conference (ICSSC), Ottawa, Canada, Sep. 2012.

${ }^{2}$ Casini. E, De Gaudenzi. R and Ginesi. A,“DVB-S2 modem algorithms design and performance over typical satellite channels," International Journal of Satellite Communications and Networking, Vol. 22, 2004, pp. 281-318.

${ }^{3}$ Gilabert. P. L, Montoro, G, Bertran. E, "On the Wiener and Hammerstein Predistortion," Proceedings of APMC, Vol. 2 , 2005, pp.1191-1194.
}

\footnotetext{
${ }^{4}$ Ding. G, Zhou. G.T, Morgan. D. R, Ma. Z,. Kenney. J. S, Kim and J, Giardina. C, R “A robust digital baseband predistorter constructed using memory polynomial," IEEE Transactions on Communications, Vol. 52, No. 1, 2004, pp. 159-165.

${ }^{5}$ Colavolpe. G and Piemontese. A,"Novel SISO Detection Algorithms for Nonlinear Satellite Channels" Proceedings of IEEE Global Telecommunications Conference, Jan. 2011, pp. 1-5.
}

${ }^{6}$ Raich. R, Hua. Q and Zhou, G. T, “Orthogonal polynomials for power amplifier modelling and predistorter design,” IEEE Transactions on Vehicular Technology, Vol. 53, No 5, 2004.

${ }^{7}$ Benedetto.S and Biglieri. E, "Nonlinear equalization of digital satellite channels," IEEE Journal on Selected Areas in Communications, Vol. 1, Jan. 1983, pp. 57-62

\footnotetext{
${ }^{8}$ Bassam. S. A, Chen. W, Helaoui. M, Ghannouchi. F. M,and, Feng. Z, "Linearization of Concurrent Dual-Band Power Amplifier Based on 2D-DPD Technique," IEEE Microwave and Wireless Components Letters, Vol. 21, No 12, Art. no. 6051495, 2011, pp. 685-687.

${ }^{9}$ Beidas. B and Seshadri. R, “Analysis and Compensation for Nonlinear Interference of Two High-Order Modulation Carriers over Satellite Link" IEEE Transactions on Communications, Vol. 58, No. 6, June 2010

${ }^{10}$ B. F. Beidas, "Intermodulation Distortion in Multicarrier Satellite Systems: Analysis and Turbo Volterra Equalization", IEEE Transactions on Communications. Vol. 59, No. 6, June 2011, pp. 1580-1590.
} 